This item was submitted to Loughborough's Research Repository by the author.

Items in Figshare are protected by copyright, with all rights reserved, unless otherwise indicated.

\title{
Systematic design, generation, and application of synthetic datasets for flow cytometry
}

PLEASE CITE THE PUBLISHED VERSION

https://doi.org/10.5731/pdajpst.2021.012659

PUBLISHER

Parenteral Drug Association, Inc.

VERSION

AM (Accepted Manuscript)

PUBLISHER STATEMENT

Reproduced with kind permission of the publisher

LICENCE

CC BY-NC-ND 4.0

\section{REPOSITORY RECORD}

Cheung, Melissa, Jonathan J Campbell, Robert J. Thomas, Julian Braybrook, and Jon Petzing. 2022. "Systematic Design, Generation, and Application of Synthetic Datasets for Flow Cytometry". Loughborough University. https://hdl.handle.net/2134/18420812.v1. 
PDA Journal

of Pharmaceutical Science and Technology

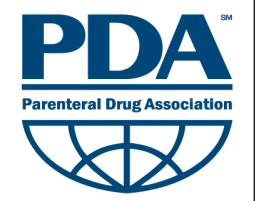

\section{Systematic design, generation, and application of synthetic datasets for flow cytometry}

Melissa Cheung, Jonathan J Campbell, Robert J Thomas, et al.

PDA Journal of Pharmaceutical Science and Technology 2022,

Access the most recent version at doi:10.5731/pdajpst.2021.012659 
4 Systematic design, generation, and application of synthetic datasets for flow 5 cytometry

7 Melissa Cheung ${ }^{1}$, Jonathan J. Campbell ${ }^{2}$, Robert J. Thomas ${ }^{1}$, Julian Braybrook ${ }^{2}$ and Jon 8 Petzing $^{1}$

$10{ }^{1}$ Centre for Biological Engineering, Loughborough University, Loughborough,

11 Leicestershire, UK

$12{ }^{2}$ National Measurement Laboratory, LGC, Teddington, UK.

13

$14 *$ Corresponding author

15 Email: M.Cheung@lboro.ac.uk

16 


\section{Abstract}

18 Application of synthetic datasets in training and validation of analysis tools have led to

19 improvements in many decision-making tasks in a range of domains from computer vision to

20 digital pathology. Synthetic datasets overcome the constraints of real-world datasets, namely

21 difficulties in collection and labelling, expense, time and privacy concerns.

22 In flow cytometry, real cell-based datasets are limited by properties such as size, number of

23 parameters, distance between cell populations and distributions, and are often focused on a

24 narrow range of disease or cell types. Researchers in some cases have designed these desired

25 properties into synthetic datasets, however operators have implemented them in inconsistent

26 approaches and there is a scarcity of publicly available, high-quality synthetic datasets.

27 In this research, we propose a method to systematically design and generate flow cytometry

28 synthetic datasets with highly controlled characteristics. We demonstrate the generation of

29 two-cluster synthetic datasets with specific degrees of separation between cell populations,

30 and of non-normal distributions with increasing levels of skewness and orientations of skew

31 pairs. We apply our synthetic datasets to test the performance of a popular automated cell

32 populations identification software, SPADE3, and define the region where the software

33 performance decreases as the clusters get closer together.

34 Application of the synthetic skewed dataset suggests the software is capable of processing

35 non-normal data. We calculate the classification accuracy of SPADE3 with robustness not

36 achievable with real-world datasets. Our approach aims to advance research towards

37 generation of high-quality synthetic flow cytometry datasets, and to increase their awareness

38 among the community.

39 The synthetic datasets can be utilised in benchmarking studies that critically evaluate cell

40 population identification tools and help illustrate potential digital platform inconsistencies. 
41 These datasets have the potential to improve cell characterisation workflows that integrate

42 automated analysis in clinical diagnostics and cell therapy manufacturing.

43

44 Keywords: flow cytometry, synthetic datasets, clusters, separation, skew, accuracy,

45 repeatability.

46 


\section{$47 \quad 1$ Introduction}

48 An important challenge in manufacturing of emerging therapies is the need to develop and

49 satisfy appropriate regulatory standards. To support advanced analytics, toolsets are required

50 to establish quality assurance, such as data reference sets for data analysis, presentation, and

51 interpretation (1).

52 Synthetic datasets are datasets generated by computer simulation, rather than collected

53 through real world observations or experiments. These datasets are often created from

54 mathematical models that approximate aspects of real-world data. Synthetic datasets can be

55 referred to as 'simulated', 'artificial', 'mock', 'toy', and more colloquially, 'dummy data'.

56 In unsupervised machine learning, well known 'toy' datasets used to compare different

57 clustering algorithms include clusters in the shape of two rings, crescent moons, spirals, and

58 data with no structures (2). Sophisticated synthetic datasets include urban street images

59 applied to object detection for autonomous driving $(3,4)$, and household objects images for

60 object detection in the field of robotic manipulation (5). In medical imaging fields, synthetic

61 datasets generated based on real images (e.g. magnetic resonance imaging (MRI),

62 mammography, and whole-slide histopathology datasets) have demonstrated utility within

63 computer-aided detection or computer-aided diagnosis systems, and may be useful for

64 educational purposes and in quality control (6-8).

65 Further strategies to generate artificial datasets have made use of data augmentation methods

66 (9). Similarly, in flow cytometry, cell subsets from real samples can be selected and

67 computationally mixed in a copy-and-paste strategy with other real or synthetic cell

68 populations, to create augmented and reprocessed 'semi-synthetic' datasets (10). 
70 Real data are generally required during the development of computational analysis tools to

71 provide means for training and validation, as well as potential decision-making reasons. An

72 analysis tool implies any method that performs detection, recognition, identification,

73 classification, tracking, prediction, or any other function that enables subsequent decision

74 making. Real data also play an important role in benchmarking studies that evaluate the

75 performance of these tools.

76 The key advantage of real data is that the information contains true characteristics of a

77 biological system. Albeit biological systems are very large and have complex signalling

78 pathway interactions between multiple cellular and molecular components. Real data have

79 various limitations that necessitates the creation of synthetic datasets to overcome them.

80 Being mathematical models, synthetic datasets can reduce the complexity of real data to gain

81 insight into how they are processed by computational analysis tools that have hidden 'black

82 box' algorithms.

83 A disadvantage of synthetic datasets is the issue of how accurately they represent the real

84 data. The purpose of synthetic datasets is to simplify real data, which requires assumptions to

85 be made and boundary conditions to be set around it, hence it is impossible to produce a

86 faithful replication of the real data. Whilst it may not be necessary to capture all the

87 complexities and features of real data in the design of synthetic ones in order to establish their

88 utility and credibility, there is potentially an expectation from users that higher complexity

89 equates to higher quality, leading to a lack of acceptance of synthetic data amongst the

90 biomedical community.

91 Often, real datasets with predetermined criteria are difficult to collect because of limited

92 availability at certain conditions and time periods. Synthetic data can be designed to mirror

93 existing real data and further optimise the dataset by including rare cases and those at 
94 extreme conditions, thereby enhancing the realistic range of features or parameters.

95 Additionally, a high level of control is potentially achievable with synthetic datasets, where

96 designers can quickly change one factor at a time or build up layers of complexity through

97 controlled addition of factors.

98 Once collected, a major shortcoming of real data is the laborious and time-consuming task of

99 labelling observations with meaningful information (e.g. healthy vs abnormal) performed by

100 experienced personnel. Synthetic datasets can be designed with the labels inherent in the data,

101 side-stepping this task. The desired property of the synthetic data is also known and can be

102 applied in performance assessment of analysis tools.

103 A drawback of large-scale real datasets is that they sometimes take a large amount of time to

104 acquire (particularly true concerning collection of rare events). An equivalent large-scale,

105 complex synthetic dataset may also require a large amount of computing time to generate,

106 however this problem is negated as computers become faster.

107 Further benefits of synthetic data are: the potential lower costs associated with use of a

108 modern computer rather than expensive technical equipment, reagents and raw materials; the

109 reproducibility of computer code; and the absence of personal data which means that the

110 processing of synthetic data does not have the same privacy concerns and legal compliance

111 requirements as that of real data (11).

112 In flow cytometry, synthetic datasets usually aim to mimic the properties of real cell

113 populations. The properties of these randomly generated datasets range from simple two-

114 dimensional datasets with four clusters (12), to up to 30 populations in 35 dimensions (13).

115 The statistical distributions of synthetic clusters vary from normal (Gaussian), to non-normal

116 generated from mixtures of several Gaussians, and skewed (14-16). Simulated background

117 noise also features $(17,18)$. Prior synthetic work approaches, however, have not explored 
118 other possible characteristics specifically such as distance between clusters (both standard

119 and rare), which is modelled in real data through the comparison of median fluorescence

120 intensities between a stained and an unstained population in terms of population widths or

121 standard deviations, in order to estimate the relative brightness of a fluorophore $(19,20)$.

122 Moreover, in a somewhat fragmented space, there is reason to apply systematic design on

123 existing properties (such as the skewness of clusters) to optimise the coverage of

124 characteristics.

125 Evaluation of developers' own tools using internally generated synthetic datasets is

126 inherently biased, therefore external and independent testing is a prerequisite for software

127 credibility in the clinical and biomanufacturing communities. Benchmarking datasets are

128 used in independent studies to compare software performance, however, existing studies

129 performed have solely relied on experimental data toolsets and have not used synthetic

130 datasets $(21,22)$. This may be related to a limited amount of synthetic datasets available

131 within public flow cytometry repositories for the community to use (23). Software

132 benchmarking studies holds similarities to other quality assurance methods, such as those

133 applied in proficiency testing defined in ISO 13528:2005 (24), and similar statistical methods

134 can be used to evaluate software output performances in an external and independent manner.

135 When using real datasets as the test material, determination of the software performance is

136 achieved through comparison of software results against an estimate of the true value. This

137 value is assigned through a choice between 1) formulation, 2) cellular certified reference

138 materials (of which very few exist for flow cytometry) (25), 3) manually gated analysis from

139 one expert, 4) consensus manual analysis results from a group of experts, or 5) consensus

140 values from participant results. Possible bias from the results of experts or participants

141 reduces the robustness of the test. Synthetic datasets can be used adjacent to certified

142 reference materials with potential benefit. 
144 In this research we propose the use of synthetic datasets for benchmarking unsupervised

145 learning automated flow cytometry data analysis software of which there are a large array of

146 options available to the data analyst (26). We define a description of the data characteristics

147 of flow cytometry data and demonstrate two methods to generate highly controlled,

148 systematically designed synthetic datasets with different degrees of separation between

149 clusters, and different levels of skew. We illustrate the use of our synthetic datasets using an

150 exemplar software, SPADE3 (27), and present results that allow robust calculations of

151 performance metrics not possible with real cell data. This work starts to explore the role of

152 synthetic datasets as digital reference materials and standards, and the potential regulatory

153 implications as the biomedical and biomanufacturing fields move increasingly towards using

154 automated systems, machine learning and artificial intelligence techniques. 


\section{Materials and Methods}

\section{$157 \quad 2.1 \quad$ Target characteristics for synthetic flow cytometry datasets}

158 We identified certain commonly recognised data characteristics or potential statistical

159 attributes of flow cytometry data and put forward a strategy to control and modify these

160 characteristics to create systematic scenarios for testing software (Table I). In this research

161 we targeted the separation / overlap and the skew properties in our simulation studies because

162 these had not been addressed in previous work and/or the designs had not been approached in

163 a systematic way. In order to focus on these properties, non-target characteristics such as the

164 number of clusters, number of datapoints, and number of dimensions were kept constant, and

165 noise was excluded in our simulations.

\section{$166 \quad 2.2$ Description of the Separation Index}

167 The separation index (SI) is used throughout this research to define the distance between 168 clusters. The SI measures the magnitude of the gap between a pair of clusters based on the 169 upper and lower percentiles of the two clusters (28). In the one-dimensional example (Figure

170 1), the SI can be summarised as Eq. 1:

$$
S I=\frac{L_{2}(\alpha / 2)-U_{1}(\alpha / 2)}{U_{2}(\alpha / 2)-L_{1}(\alpha / 2)} \quad \text { Eq. } 1
$$

172 where $L_{i}(\alpha / 2)$ and $U_{i}(\alpha / 2)$ are the sample lower and upper $(\alpha / 2)$ quantiles of cluster $i$. The 173 interpretation of the SI is relatively straight forward, the range is $[-0.999,+0.999]$ with values

174 approaching +1 indicating increasing separation, SI of 0 indicating clusters touching, and SI

175 approaching -1 indicating total overlap. In practice, our working range for the SI was [-0.3,

$176+0.3]$. These limits were defined because at a SI of +0.3 clusters were already very well

177 separated, and at a SI of -0.3 clusters appeared well overlapped or merged. 


\section{$179 \quad 2.3 \quad$ Hardware and software}

180 Dataset generation and analysis was run on a 64-bit Windows 10 operating system with a

$1813.00 \mathrm{GHz}$ processor and $64 \mathrm{~GB}$ of RAM. Computational tools used are listed in Table II.

182 Throughout this paper, we use regular type to refer to software or computing environments,

183 italics for packages, and monospace font to designate functions.

\section{$184 \quad 2.4 \quad$ Synthetic datasets}

185 The concept of creating artificial, computer-generated flow cytometry datasets is essentially

186 random number generation, with numbers typically drawn from a normal distribution. Other

187 probability distributions are available e.g. binomial, exponential, Poisson, Student's t, etc. If

188 flow cytometry data are considered as mixtures of subpopulations of a heterogenous sample,

189 then the generation of synthetic data is a process of creating a mixture of random clusters.

\subsubsection{Separation dataset generation}

192 We designed a library of two-cluster synthetic datasets in two dimensions with 1,000

193 datapoints per cluster as an exemplar size of cell populations in real flow cytometry data,

194 with different degrees of separation between neighbouring clusters ranging from wellseparated to merged. The datasets were prepared using the $\mathrm{R}$ clusterGeneration package, with

196 SI values ranging from $[-0.3,+0.3]$ at 0.05 intervals. Nine random normally distributed

197 cluster replicates were generated at each SI value. Covariance matrices were randomly

198 generated from eigenvalues between 1 and 5 to give a variability in the diameter and shape of

199 clusters that is similar to those seen in real flow cytometry data. These parameters produced

200 clusters with known separation, but which were random in their elliptical shape attribute.

201 Datasets were converted to FCS3.1 format using the R package flowCore. 


\section{$203 \quad 2.4 .2$ Skew dataset generation}

204 We designed a library of two-cluster synthetic datasets in two dimensions with 1,000

205 datapoints per cluster, with different levels of skew and skew-direction pairs. Single skew

206 clusters were prepared with the package $s n$, of which the $\alpha$ parameter regulates asymmetry

207 (29). Likewise random cluster replicates were generated at each skew direction (left and

208 right) along the $\mathrm{x}$-axis in addition to each level of skew input $\alpha$ values between 2.5 to 10 , at

209 intervals of 2.5. Applying the skewing $\alpha$ parameter causes the diameter of the elliptical

210 cluster to reduce along the x-axis. To compensate for this, clusters were elongated to obtain a

211 pre-skew diameter using the package rescale. The skewness of the clusters before and after

212 rescaling were identical (measured using the package psych) determined by the asymmetry

213 around the mean remaining unchanged (Figure 2A). Two clusters were joined together, and

214 one cluster shifted further away from the other through vector arithmetic operations in $\mathrm{R}$

215 (Figure 2B). The distance between two clusters was measured with the clusterGeneration

216 package, datasets with a SI value between -0.25 and -0.15 were selected for further

217 processing. Files were converted to FCS3.1 standard using flowCore and visualised within

218 FlowJo software.

\subsection{Real datasets}

221 All material was obtained with the approval of and in accordance with the respective Ethics

222 Committees of Loughborough University and LGC, and under jurisdiction of the Human

223 Tissue Authority.

\section{$224 \quad$ 2.5.1 Real cell PBMC dataset 1}

225 Fresh whole blood from healthy donors (Cambridge Bioscience, UK) was processed using

226 Ficoll-Paque (Fisher) to isolate the buffy coat layer containing peripheral blood mononuclear 
227 cells (PBMCs). Cells were single-stained separately with CD4-PerCP-Vio700, CD45RO-

228 APC-Vio770, and CCR7-VioBlue (all from Miltenyi Biotech). Data were acquired using BD

229 FACSCantoII cytometer equipped with 3 lasers $(405 \mathrm{~nm} / 30 \mathrm{~mW}, 488 \mathrm{~nm} / 20 \mathrm{~mW}, 633 \mathrm{~nm} /$

$23017 \mathrm{~mW}) .100,000$ cell events were collected.

$231 \quad$ 2.5.2 Real cell PBMC dataset 2

232 PBMCs (LGC, UK) were stained with CD3-BB515, CD4-BB700, CD45RA-BV786 (all from

233 BD Biosciences), and live/dead fixable aqua dead cell stain (Invitrogen). Data were acquired

234 using a BD LSRFortessa cell analyser equipped with four lasers (355nm $/ 20 \mathrm{~mW}, 405 \mathrm{~nm} /$

$23550 \mathrm{~mW}, 488 \mathrm{~nm} / 50 \mathrm{~mW}, 640 \mathrm{~nm} / 40 \mathrm{~mW}) .200,000$ cell events were collected. Single-stained

236 beads and fluorescence-minus-one controls were used to calculate compensation.

\subsection{SPADE3 analysis of synthetic datasets}

239 SPADE3 was run within Matlab R2019a. Each FCS file was run separately. User input

240 parameters that were selected were: overlapping markers used for SPADE tree $=\mathrm{CH} 1, \mathrm{CH} 2$;

241 ignore compensation; no transformation. All other settings were left as default values (local

242 density neighbourhood size $=5$, local density approximation factor $=1.5$, maximum

243 allowable cells $=50,000$, outlier density $=1$, target density $=20,000$ cells, algorithm $=\mathrm{K}-$

244 means, number of desired clusters $=100)$.

\section{$246 \quad 2.7 \quad$ Statistics and performance metrics}

247 Methods used for statistical analysis included the mean, standard deviation, coefficient of

248 variation, and metrics derived from the confusion matrix as shown in Table III.

249 The performances of software runs were calculated using Eq. 2 and Eq. 3: 


$$
\text { Population percentage difference }(\%)=\frac{|A-B|}{\text { Total population }} \times 100
$$

250 where $A$ is the software output count, and $B$ is the reference value defined here as the known

251 count of cluster 1 (1,000 events) which was designed inherently in the dataset.

252 As in binary classification (30), here a true positive (a 'hit') is defined as the correct SPADE3

253 assignment of a target cell to its reference target population set during cluster generation.

254 Events in cluster 1 of the synthetic datasets were arbitrarily selected as the 'target' cases.

255 SPADE3 assignment of a non-target cell to its non-target population is a true negative.

256 Misclassification of a non-target cell to a target population is a false positive, and

257 misclassification of a target cell to a non-target population is a false negative (a 'miss'). The

258 evaluation metrics calculated from the confusion matrix include the accuracy, precision,

259 recall and F1 measure, and are defined in Eq. 4 to Eq. 7 (31). We compared the individual

260 cell assignments to a cluster predicted from SPADE3 with the reference cell assignments,

261 using the R package caret.

$$
\text { Accuracy }=\frac{\text { True positive }+ \text { True negative }}{\text { Total population }}
$$

$$
\text { Precision }=\frac{\text { True positive }}{\text { True positive }+ \text { False positive }}
$$

$$
\text { Recall }=\frac{\text { True positive }}{\text { True positive }+ \text { False negative }} \quad \text { Eq. } 6
$$




$$
F 1=2 \times \frac{\text { Precision } \times \text { Recall }}{\text { Precision }+ \text { Recall }}
$$

Eq. 7

266 


\section{Discussion}

268 In flow cytometry, data typically contain cell populations that are positive or negative for a

269 marker of interest. The distance between the positive and negative cell populations is

270 variable, ranging from well resolved to merged. Multiple factors affect this separation,

271 including but not limited to biological attributes (the level of marker expression, affinity and

272 avidity of antibody binding, the number of antibody bound per cell) and assay variables

273 (antibody panel design and concentrations used in the staining process, fluorophore

274 brightness and dye stability, sensitivity and resolution of the detectors). This separation

275 directly impacts the accuracy and precision of manual data analysis, with significantly higher

276 technical variation seen in poorly resolved populations compared with clearly defined cell

277 populations in human peripheral blood (32).

\section{$279 \quad 3.1 \quad$ Distance between clusters}

280 To simulate flow cytometry data with different distances between a positive and a negative 281 population we generated a normally distributed synthetic two-cluster dataset with different 282 degrees of separation between clusters. For comparison, we measured the SI between two 283 clusters in a real cell dataset. The PBMCs dataset 1 contained negatively and positively

284 stained populations in each fluorescent channel. These subpopulations were separated using 285 the automated cell population identification software SPADE3 (27). Then the magnitude of 286 the gap between pairs of real cell clusters along each channel was measured using the sepIndex function in the clusterGeneration package. We observed similar SI values

288 between the real-world positive and negatively stained cell populations and the synthetic 289 clusters, within the range of -0.3 and +0.2 (Figure 3 ). These results show our method defined

290 here for generating synthetic flow cytometry datasets is able to successfully simulate the

291 distance parameters seen between clusters in a real example of flow cytometry data. 
292 Individual cluster statistics can potentially vary by a small amount, however at this stage of

293 the research this was not a focus of this study.

\section{$295 \quad 3.2$ Clusters with non-normal distributions}

296 The synthetic datasets generated in Section 3.1 contain clusters following a normal

297 distribution, visualised as symmetrical bell curves for univariate data or symmetrical circles

298 and ellipses in scatterplots for multivariate data. However, real flow cytometry data consist of

299 cell population clusters that follow a normal distribution as well as those that display non-

300 normal distributions. The exact distribution along a marker channel is difficult to predict and

301 may depend on the state of the cell along a differentiation pathway. For example, a stable

302 haematopoietic stem cell population may display a normal distribution of CD34+ expression

303 that transitions to a non-normal distribution during cell differentiation as CD34 expression

304 decreases (33).

305 Non-normal data are characterised by asymmetry around the sample mean. These cell

306 populations can display positive (right) skew or negative (left) skew. The skewness can be

307 estimated using the adjusted Fisher-Pearson coefficient of skewness ( $p$ value) (34), where a

308 normal distribution has a skewness value of $p=0$, a positive skewness value indicates a tail

309 pointing to the right, and a negative skewness value indicates a tail pointing to the left. The

310 further away the value is from 0 , the greater the skew and typically the longer the tail.

311 There are different strategies to generate synthetic flow cytometry datasets with skewed cell

312 populations. One method is to create multiple Gaussian distributions that can then be merged

313 together to form an overall distribution with the desired skew. This strategy has been used

314 previously to create synthetic data with non-convex shapes $(15,16)$. This method may require

315 many rounds of trial and error. To avoid this shortcoming, here we developed and tested a 
316 different method using the $s n$ R package to generate random clusters with multivariate skew-

317 normal distributions (29).

318 The multivariate skew normal distribution extends the class of normal distribution (defined

319 through a mean vector and covariance matrix) by the addition of a skew parameter.

320 A comparison of the skewed clusters generated from computer simulation against real cell

321 populations from the PBMCs dataset 2 demonstrates that the synthetic and real cases are

322 comparable (Figure 4). This result shows that the simulated data we generated is a realistic

323 model of both positive and negative skew observed in real flow cytometry cell populations,

324 and therefore has biological relevance. Thus, the synthetic dataset can be reliably used to gain

325 understanding on how automated software responds to skewed flow cytometry data, with the

326 additional benefit of the ability to systematically control the strength of the skew as well as

327 the absolute cell number.

328 The strategy we devised to create a dataset with multiple skewed clusters was to generate

329 individual clusters in parts then combine them together to form one whole dataset. The gap

330 between the clusters can be controlled by shifting one cluster closer or further away to the

331 other through vector arithmetic operations, with the SI being measured after the clusters were

332 combined. With skewed clusters, a new level of complexity is introduced compared to

333 normally distributed clusters, because assuming the skew is introduced only in one parameter,

334 each cluster can be left-skewed or right-skewed. Thus, the possible permutations of pairs of

335 skewed clusters in a two-cluster dataset increases from one to three. In this paper we refer to

336 these combinations as: head-to-head, head-to-tail (this is the same orientation as tail-to-head),

337 and tail-to-tail (Figure 5). 
$339 \quad 3.3$ Application of synthetic datasets to a cell population identification software

340 To demonstrate the efficacy of the synthetic datasets, they were passed through an exemplar

341 software, SPADE, in order to illustrate how synthetic data can reveal limitations of

342 automated software, and can provide a deeper understanding of the inner workings of 'black

343 box' algorithms in a way that real cell datasets are unable to.

344 SPADE, named for spanning-tree progression analysis for density-normalised events, is a

345 widely applied software package that uses automated down-sampling, clustering and

346 minimum spanning tree construction to aid analysis of high-dimensional flow cytometry data

347 (35). There are two versions of SPADE with different algorithms. The original SPADE1

348 applies a stochastic down-sampling algorithm paired with an agglomerative hierarchical

349 clustering algorithm that produces different outputs when run on the same data. This specific

350 issue of reproducibility in SPADE1 was subsequently resolved in SPADE3 by removing the

351 stochastic algorithms and replacing them with deterministic ones (27). In addition, a tree

352 partitioning function was introduced to assist interpretation of the outputs.

353 Here, we used the Matlab implementation of SPADE3 to process our synthetic datasets using

354 default parameters (as described in section 2.6). We used the auto tree partitioning tool to

355 split the spanning tree into two populations, then compared the population number to our

356 known reference value, which was 1,000 cells per cluster, or $50 \%$ of total cells events, for

357 both the separation and skew datasets.

358 For the separation dataset, the absolute difference in cell count of each cluster between the

359 software output and the reference value was calculated for each SI condition. The results

360 show that the accuracy and precision of SPADE3 decreased as the SI decreased from +0.3 to

$361-0.3$, with performance deteriorating noticeably at a SI value of -0.2 and below (Figure 6). 
362 These results were to be expected, because defining the boundary between one cluster and

363 another becomes progressively more difficult as clusters get closer together.

364 The benefit of applying the synthetic datasets to test software such as SPADE3 was the

365 ability to quantify for the first time the SI value where the software began to lose

366 performance. The high level of control in designing the gap between cell populations within

367 the synthetic datasets would have been very difficult to achieve with real cell data.

368 Furthermore, since the absolute counts and frequencies of each cell population was known in

369 the synthetic dataset, the evaluation of the software was based on robust absolute traceable

370 figures, and did not rely on comparison with a manually gated reference subpopulation count,

371 which has already been shown to be operator dependent and potentially biased $(36,37)$.

372 In the design of the skew dataset, a constant SI value of -0.2 between clusters was chosen

373 because it fell in the critical region where the SPADE3 software began to deteriorate. The

374 skew dataset was processed through SPADE3, then the difference in cell population

375 percentage of the cluster between the SPADE3 output and the reference value was calculated

376 for each skew condition and cluster pair orientation. We found that, for each cluster pair

377 orientation, increasing the level of skewness in the clusters had no effect on the accuracy and

378 precision of SPADE3. However, at each level of skewness, SPADE3 was able to partition the

379 two clusters with improved performance when the orientation was tail-to-tail, followed by

380 head-to-tail and finally head-to-head (Figure 7).

382 This pattern of performance appeared to correlate with the density of points between the two

383 clusters. The skew dataset was planned with skewness and skew orientation among the

384 variable design factors, and the separation between clusters as constant factors. The

385 systematic way this skew dataset was designed allowed for the pattern of behaviour of 
386 SPADE3 to become apparent. This finding suggests the SPADE3 algorithm is well suited to

387 analysis of skewed data albeit with a performance bias and sensitivity depending on skewed

388 cluster orientation. This may not be the case for other algorithms that use different clustering

389 techniques, in particular those that use a Gaussian mixture model-based clustering approach.

390 Further work to investigate this in a software comparison study is warranted.

391

$392 \quad 3.4$ Assessment of software performance based on synthetic data

393 One of the benefits of synthetic data is that, as well as 'true' population counts and

394 frequencies, an estimate of the true membership of a cell to its cluster is known a priori. This

395 is not the case with real cell data, where membership of a cell to a population is estimated by

396 an analyst performing manual gating. Here, we demonstrate the evaluation of robust

397 performance metrics of SPADE3 runs on synthetic datasets using confusion matrix analysis.

398 Each cell event in the synthetic dataset was pre-assigned a cluster membership on generation.

399 These cluster memberships were withheld for the SPADE3 analyses. After running the

400 datasets through SPADE3, the software predictions of cluster memberships for all 2,000 cell

401 events were compared with the reference cluster memberships using the $\mathrm{R}$ caret package.

402 Events in cluster 1 were arbitrarily assigned as positive cases.

403 The results from the SPADE3 analysis of the synthetic separation dataset (Table IV) showed 404 a classification accuracy (Eq. 4) greater than $90 \%$ with SI values of -0.1 or greater. Accuracy

405 fell to $86 \%$ and $43 \%$ at SI values of -0.2 and -0.3 respectively (Figure $8 \mathrm{~A}$ ).

406 The same pattern appeared with precision, also called positive predictive value (Eq. 5) with

407 values greater than $90 \%$ at SI values of -0.1 or larger, then falling to $81 \%$ and $50 \%$ at SI

408 values of -0.2 and -0.3 respectively (Figure $8 \mathrm{~B}$ ). 
409 The recall metric that measures the rate of true positives identified (Eq. 6) gave perfect scores

410 of $100 \%$ at SI of +0.3 , scored greater than $90 \%$ at SI values of -0.2 or greater, and

411 deteriorated to $46 \%$ with SI of -0.3 (Figure 8 C).

412 The F1 score (Eq. 7) was calculated from the precision and accuracy to give the overall

413 accuracy of SPADE3. The F1 score remained above $90 \%$ for SI values greater than -0.1 , then

414 fell to $85 \%$ at a SI value of -0.2 and reduced further to $47 \%$ at a SI value of -0.3 (Figure $8 D$ ).

415 The results from the classification analysis reinforce the finding that SPADE3 performs

416 strongly when clusters are well-separated, but the accuracy decreases as clusters approach a

417 SI value of -0.2 and falls to below $50 \%$ when processing overlapping clusters with SI values

418 of -0.3. Our application of this synthetic flow cytometry dataset in this instance has helped to

419 illustrate good performance characteristics and ranges, but also limitations of SPADE3 with

420 respect to cluster separation both with normal and skewed cluster probability distributions. 


\section{Conclusion}

424 In this article we have introduced a systematic method of designing and generating synthetic

425 flow cytometry datasets, with specific focus on control of the distance between clusters and

426 the probability distributions of events within clusters. We applied our computer-generated

427 flow cytometry datasets to an automated data analysis software, SPADE3, and have shown

428 that the synthetic datasets are capable of critically assessing the quality of the software

429 outputs and hence the software performance. In addition, we have given an example of 430 quantifying performance assessment using synthetic datasets that is robust compared with 431 using real-world datasets.

433 The systematic approach we have implemented to produce flow cytometry datasets is straight 434 forward to execute computationally, but would be complicated to achieve experimentally due 435 to uncontrollable external sources of variation within real cell datasets, thus synthetic datasets

436 here overcome the limitations of acquiring real datasets. The synthetic datasets have the same 437 range of data properties as their biological equivalents and can serve as credible substitutes

438 for real flow cytometry datasets for the testing of automated cell population identification 439 software.

441 It is noted here that in most cases algorithms that underpin flow cytometry analysis software 442 have been previously published, but it is (at times) inherently opaque implementation of 443 algorithms in executable code that whilst allows understanding of inputs and outputs, does 444 not allow a full understanding of the data transfer functions. Application of our synthetic 445 datasets to an automated cell population identification software such as SPADE3 can 
446 therefore help users understand how the underlying 'black box' algorithm works. Here we

447 have identified the regions where SPADE3 begins to lose performance, specifically where

448 two clusters are located at a SI value of -0.2 or less. Our results suggest that SPADE3 is not

449 specifically affected by the probability distributions of data, but is more sensitive to the

450 relative density of data points between two clusters. Findings such as these can provide

451 guidance to users on software selection when having to contend with large array of potential

452 software solutions (26) (i.e. in this exemplar whether SPADE3 would be an appropriate tool

453 of choice for automated analysis of real data containing heavy overlapping of clusters), and

454 then help to understand the validity of their automated analysis outputs.

456 A further benefit of synthetic datasets was apparent when we assessed the performance of

457 SPADE3 using the metrics calculated from the confusion matrix. This classification analysis

458 relied on comparison of software predictions with known 'true' conditions hence an absolute

459 analysis. In the synthetic dataset, an estimate of the true assignment of cells to clusters was

460 designed into the data, making this analysis relatively rapid and robust. With real data, the

461 assignment of cells to subpopulations must first be manually determined (often with potential

462 difficulty and error), then the dataset labelled, before a confusion matrix can be calculated.

463 These additional steps are time-consuming and prone to error. The variability observed in 464 manually gated datasets means either the analysis from a single expert must be taken as the

465 best estimate of the 'true value', or a pooled manual analysis from a group of experts is used.

466 The first option risks bias, and the second is dependent on the precision and accuracy of the

467 group. In both instances, it is difficult for the final analysis to be as robust compared with

468 synthetic datasets. 
469 There are also a few disadvantages with synthetic datasets. The datasets are built on

470 assumptions of real data. These approximations are based on mathematical models and have

471 limits. Although the aim is to create synthetic datasets that are as realistic as possible, there

472 may be features missing as a function of boundary conditions and design assumptions.

474 Further investigations on flow cytometry synthetic datasets will follow two main directions.

475 The first is the extension of the work to generate datasets with controls on other flow

476 cytometry data properties identified in Table I, and more complex datasets with multiple

477 controlled factors. We are currently developing and optimising synthetic datasets with rare

478 cell populations with and without skewed distributions, and noise characteristics. The second

479 area is the comparison of software performance across multiple platforms when challenged

480 with synthetic datasets targeting flow cytometry automated data analysis software that

481 employ various clustering algorithms such as K-means, hierarchical, partition, density-based,

482 model-based, spectral clustering and self-organising maps.

484 Besides being a benchmarking tool for software developers, possible further applications of

485 synthetic datasets include their use as educational and training tools for manual gating, as part

486 of external quality assessment (EQA) and proficiency testing schemes. In addition, as cell

487 identification and quantification in medical diagnostics and cell therapy/ regenerative

488 medicines manufacturing fields move increasingly towards automated machine learning and

489 artificial intelligence techniques, it is likely that synthetic datasets will have important

490 regulatory applications as digital reference materials and standards, as well as potential

491 regulatory implications. 


\section{Acknowledgements}

494 The authors would like to thank Lydia Beeken and Dr Ben Diffey from the Centre for

495 Biological Engineering, Loughborough University, and Dr Shiqiu Xiong at LGC, for their

496 help with acquiring real flow cytometry datasets.

497

498 Conflict of Interest Declaration

499 The authors declare that they have no competing interests.

500 


\section{References}

502 (1) Phillips, W.; Medcalf, N.; Dalgarno, K.; Makatoris, H.; Sharples, S.; Srai, J.; Hourd, P.; Kapletia, D. Redistributed Manufacturing in Healthcare: Creating New Value through Disruptive Innovation; 2019.

Pedregosa, F.; Varoquaux, G.; Gramfort, A.; Michel, V.; Thirion, B.; Grisel, O.; Blondel, M.; Prettenhofer, P.; Weiss, R.; Dubourg, V.; Vanderplas, J.; Passos, A.; Cournapeau, D.; Brucher, M.; Perrot, M.; Duchesnay, É. Scikit-Learn: Machine Learning in Python. J. Mach. Learn. Res. 2011, 12 (Oct), 2825-2830.

Ros, G.; Sellart, L.; Materzynska, J.; Vazquez, D.; Lopez, A. M. The SYNTHIA Dataset: A Large Collection of Synthetic Images for Semantic Segmentation of Urban Scenes. Proc. IEEE Comput. Soc. Conf. Comput. Vis. Pattern Recognit. 2016, 32343243. https://doi.org/10.1109/CVPR.2016.352.

(4) Wrenninge, M.; Unger, J. Synscapes: A Photorealistic Synthetic Dataset for Street Scene Parsing. arXiv Preprint. 2018. https://arxiv.org/abs/1810.08705 (accessed 202011-20)

(5) Tremblay, J.; To, T.; Birchfield, S. Falling Things: A Synthetic Dataset for 3D Object Detection and Pose Estimation. IEEE Comput. Soc. Conf. Comput. Vis. Pattern Recognit. Work. 2018, 2038-2041. https://doi.org/10.1109/CVPRW.2018.00275.

(6) Hagiwara, A.; Warntjes, M.; Hori, M.; Andica, C.; Nakazawa, M.; Kumamaru, K. K.; Abe, O.; Aoki, S. SyMRI of the Brain: Rapid Quantification of Relaxation Rates and Proton Density, with Synthetic MRI, Automatic Brain Segmentation, and Myelin Measurement. Invest. Radiol. 2017, 52 (10), 647-657. https://doi.org/10.1097/RLI.0000000000000365.

(7) Ratanaprasatporn, L.; Chikarmane, S. A.; Giess, C. S. Strengths and Weaknesses of Synthetic Mammography in Screening. Radiographics 2017, 37 (7), 1913-1927. https://doi.org/10.1148/rg.2017170032.

(8) Niazi, M. K. K.; Parwani, A. V.; Gurcan, M. N. Digital Pathology and Artificial Intelligence. Lancet Oncol. 2019, 20 (5), e253-e261. https://doi.org/10.1016/S14702045(19)30154-8.

(9) Perez, L.; Wang, J. The Effectiveness of Data Augmentation in Image Classification Using Deep Learning. arXiv Preprint. 2017. https://arxiv.org/abs/1712.04621 (accessed 2021-01-05).

(10) Arvaniti, E.; Claassen, M. Sensitive Detection of Rare Disease-Associated Cell Subsets via Representation Learning. Nat. Commun. 2017, 8 (1), 1-10. https://doi.org/10.1038/ncomms14825.

(11) The European Parliament and the Council of the European Union. Regulation (EU) 2016/679 of the European Parliament and of the Council of 27 April 2016 on the Protection of Natural Persons with Regard to the Processing of Personal Data and on the Free Movement of Such Data, and Repealing Directive 95/46/EC. Off. J. Eur. Union 2016, 1-88.

(12) Sugar, I. P.; Sealfon, S. C. Misty Mountain Clustering: Application to Fast Unsupervised Flow Cytometry Gating. BMC Bioinformatics 2010, 11 (1), 1-14. 
https://doi.org/10.1186/1471-2105-11-502.

544

545

546

547

548

549

550

551

552

553

554

555

556

557

558

559

560

561

562

563

564

565

566

567

568

569

570

571

572

573

574

575

576

577

578

579

580

581

582

583

584

585

(13) Samusik, N.; Good, Z.; Spitzer, M. H.; Davis, K. L.; Nolan, G. P. Automated Mapping of Phenotype Space with Single-Cell Data. Nat. Methods 2016, 13 (6), 493-496. https://doi.org/10.1038/nmeth.3863.

(14) Naim, I.; Datta, S.; Rebhahn, J.; Cavenaugh, J. S.; Mosmann, T. R.; Sharma, G. SWIFT-Scalable Clustering for Automated Identification of Rare Cell Populations in Large, High-Dimensional Flow Cytometry Datasets, Part 1: Algorithm Design. Cytom. Part A 2014, 85 (5), 408-421. https://doi.org/10.1002/cyto.a.22446.

(15) Pyne, S.; Hu, X.; Wang, K.; Rossin, E.; Lin, T.-I.; Maier, L. M.; Baecher-Allan, C.; McLachlan, G. J.; Tamayo, P.; Hafler, D. A.; De Jager, P. L.; Mesirov, J. P. Automated High-Dimensional Flow Cytometric Data Analysis. Proc. Natl. Acad. Sci. 2009, 106 (21), 8519-8524. https://doi.org/10.1073/pnas.0903028106.

(16) Ge, Y.; Sealfon, S. C. Flowpeaks: A Fast Unsupervised Clustering for Flow Cytometry Data via K-Means and Density Peak Finding. Bioinformatics 2012, 28 (15), 20522058. https://doi.org/10.1093/bioinformatics/bts300.

(17) Zare, H.; Shooshtari, P.; Gupta, A.; Brinkman, R. R. Data Reduction for Spectral Clustering to Analyze High Throughput Flow Cytometry Data. BMC Bioinformatics 2010, 11 (1), 1-16. https://doi.org/10.1186/1471-2105-11-403.

(18) Bendall, S. C.; Davis, K. L.; Amir, E. A. D.; Tadmor, M. D.; Simonds, E. F.; Chen, T. J.; Shenfeld, D. K.; Nolan, G. P.; Pe'Er, D. Single-Cell Trajectory Detection Uncovers Progression and Regulatory Coordination in Human B Cell Development. Cell 2014, 157 (3), 714-725. https://doi.org/10.1016/j.cell.2014.04.005.

(19) Bigos, M. Separation Index: An Easy-to-Use Metric for Evaluation of Different Configurations on the Same Flow Cytometer. Curr. Protoc. Cytom. 2007, 40 (1), 1-21. https://doi.org/10.1002/0471142956.cy0121s40.

(20) Telford, W. G.; Babin, S. A.; Khorev, S. V.; Rowe, S. H. Green Fiber Lasers: An Alternative to Traditional DPSS Green Lasers for Flow Cytometry. Cytom. Part A 2009, 75 (12), 1031-1039. https://doi.org/10.1002/cyto.a.20790.

(21) Aghaeepour, N.; Finak, G.; Hoos, H.; Mosmann, T. R.; Brinkman, R.; Gottardo, R.; Scheuermann, R. H.; Gottardo, R.; Scheuermann, R. H. Critical Assessment of Automated Flow Cytometry Data Analysis Techniques. Nat. Methods 2013, 10 (3), 228-238. https://doi.org/10.1038/nmeth.2365.

(22) Weber, L. M.; Robinson, M. D. Comparison of Clustering Methods for HighDimensional Single-Cell Flow and Mass Cytometry Data. Cytom. Part A 2016, 89 (12), 1084-1096. https://doi.org/10.1002/cyto.a.23030.

(23) Spidlen, J.; Breuer, K.; Rosenberg, C.; Kotecha, N.; Brinkman, R. R. FlowRepository: A Resource of Annotated Flow Cytometry Datasets Associated with Peer-Reviewed Publications. Cytom. Part A 2012, 81 (9), 727-731. https://doi.org/10.1002/cyto.a.22106.

(24) International Organization for Standardization. ISO 13528:2005 Statistical Methods for Use in Proficiency Testing by Interlaboratory Comparison; Geneva, 2005.

(25) Wang, L.; Abbasi, F.; Ornatsky, O.; Cole, K. D.; Misakian, M.; Gaigalas, A. K.; He, H. J.; Marti, G. E.; Tanner, S.; Stebbings, R. Human CD4 + Lymphocytes for Antigen 
Quantification: Characterization Using Conventional Flow Cytometry and Mass Cytometry. Cytom. Part A 2012, 81 A (7), 567-575. https://doi.org/10.1002/cyto.a.22060.

(26) Cheung, M.; Campbell, J. J.; Whitby, L.; Thomas, R. J.; Braybrook, J.; Petzing, J. N. Current Trends in Flow Cytometry Automated Data Analysis Software. Cytom. Part A 2021, 99 (10), 1007-1021. https://doi.org/10.1002/cyto.a.24320.

(27) Qiu, P. Toward Deterministic and Semiautomated SPADE Analysis. Cytom. Part A 2017, 91 (3), 281-289. https://doi.org/10.1002/cyto.a.23068.

(28) Qiu, W.; Joe, H. Separation Index and Partial Membership for Clustering. Comput. Stat. Data Anal. 2006, 50 (3), 585-603. https://doi.org/10.1016/j.csda.2004.09.009.

(29) Azzalini, A.; Capitanio, A. Statistical Applications of the Multivariate Skew Normal Distribution. J. R. Stat. Soc. Ser. B Stat. Methodol. 1999, 61 (3), 579-602. https://doi.org/10.1111/1467-9868.00194.

(30) Fleiss, J. L.; Levin, B.; Paik, M. C. Statistical Methods for Rates and Proportions, 3rd ed.; John Wiley \& Sons: Hoboken, NJ, USA, 2003.

(31) Tharwat, A. Classification Assessment Methods. Appl. Comput. Informatics 2021, 17 (1), 168-192. https://doi.org/10.1016/j.aci.2018.08.003.

(32) Burel, J. G.; Qian, Y.; Lindestam Arlehamn, C.; Weiskopf, D.; Zapardiel-Gonzalo, J.; Taplitz, R.; Gilman, R. H.; Saito, M.; de Silva, A. D.; Vijayanand, P.; Scheuermann, R. H.; Sette, A.; Peters, B. An Integrated Workflow to Assess Technical and Biological Variability of Cell Population Frequencies in Human Peripheral Blood by Flow Cytometry. J. Immunol. 2017, 198 (4), 1748-1758. https://doi.org/10.4049/jimmunol.1601750.

(33) Salati, S.; Zini, R.; Bianchi, E.; Testa, A.; Mavilio, F.; Manfredini, R.; Ferrari, S. Role of CD34 Antigen in Myeloid Differentiation of Human Hematopoietic Progenitor Cells. Stem Cells 2008, 26 (4), 950-959. https://doi.org/10.1634/stemcells.2007-0597.

(34) Joanes, D. N.; Gill, C. A. Comparing Measures of Sample Skewness and Kurtosis. J. R. Stat. Soc. Ser. D (The Stat.) 1998, 47 (1), 183-189. https://doi.org/10.1111/14679884.00122.

(35) Qiu, P.; Simonds, E. F.; Bendall, S. C.; Gibbs, K. D.; Bruggner, R. V; Linderman, M. D.; Sachs, K.; Nolan, G. P.; Plevritis, S. K. Extracting a Cellular Hierarchy from HighDimensional Cytometry Data with SPADE. Nat. Biotechnol. 2011, 29 (10), 886-893. https://doi.org/10.1038/nbt.1991.

(36) Grant, R.; Coopman, K.; Medcalf, N.; Silva-Gomes, S.; Campbell, J. J.; Kara, B.; Braybrook, J.; Petzing, J. Understanding the Contribution of Operator Measurement Variability within Flow Cytometry Data Analysis for Quality Control of Cell and Gene Therapy Manufacturing. Measurement 2020, 150, 106998. https://doi.org/10.1016/j.measurement.2019.106998.

(37) Grant, R.; Coopman, K.; Medcalf, N.; Silva-Gomes, S.; Campbell, J. J.; Kara, B.; Braybrook, J.; Petzing, J. N. Quantifying Operator Subjectivity within Flow Cytometry Data Analysis as a Source of Measurement Uncertainty and the Impact of Experience on Results. PDA J. Pharm. Sci. Technol. 2021, 75 (1), 33-47. https://doi.org/10.5731/pdajpst.2019.011213. 


\section{Downloaded from on January 18, 2022}

629 
Table I. Characteristics of flow cytometry datasets

\begin{tabular}{ll}
\hline Characteristic & Description \\
\hline $\begin{array}{l}\text { Number of clusters } \\
\text { Number of datapoints }\end{array}$ & $\begin{array}{l}\text { Number of cell subpopulations in a sample } \\
\text { Number of cell events acquired from a sample }\end{array}$ \\
\hline $\begin{array}{l}\text { Number of dimensions } \\
\text { Separation }\end{array}$ & $\begin{array}{l}\text { The scatter, fluorescent markers } \\
\text { The gap between negatively and positively stained cell populations }\end{array}$ \\
Overlap & $\begin{array}{l}\text { Poorly resolved populations that appear merged } \\
\text { Placement }\end{array}$ \\
Distribution & $\begin{array}{l}\text { The shape of the cell population, as modelled on probability distributions } \\
\text { e.g. Gaussian, Student's t, exponential, Chi-squared }\end{array}$ \\
Spread & $\begin{array}{l}\text { The variance of the cell population } \\
\text { The level of asymmetry around the mean of the cell cluster }\end{array}$ \\
Skew and kurtosis & The direction of the asymmetry \\
Orientation & Stretched out populations with long tails \\
Elongation & $\begin{array}{l}\text { Events that are excluded from analysis e.g. outliers, dead cells, debris, } \\
\text { doublets, false events detected in the region of interest. }\end{array}$ \\
\hline Noise &
\end{tabular}

632

Table II. Toolset used in this research for the generation of synthetic datasets, automated cell population identification, and performance evaluation. $\mathrm{R}$ packages in italics.

\begin{tabular}{lll}
\hline Tool & Version & Purpose in this research \\
\hline $\mathrm{R}$ & 3.5 .1 & Programming \\
\hline RStudio IDE & 1.2 & Programming environment \\
\hline Matlab & $\mathrm{R} 2019 \mathrm{a}$ & Environment for SPADE analysis \\
\hline FlowJo & 10.6 & Flow cytometry data analysis and visualisation \\
\hline SPADE & 3 & Automated analysis of synthetic datasets \\
\hline caret & $6.0-82$ & Calculate performance metrics, confusion matrix \\
\hline clusterGeneration & 1.3 .4 & Generate synthetic clusters \\
\hline flowCore & 1.48 .1 & Manipulate flow cytometry data \\
\hline psych & 1.8 .12 & Measure skew \\
\hline scales & 1.1 .0 & Scale functions for visualisation \\
\hline sn & $1.5-3$ & $\begin{array}{l}\text { Build and manipulate probability distributions of the } \\
\text { skew-normal family }\end{array}$ \\
\hline tidyverse & 1.3 .0 & Data manipulation, analysis and visualisation \\
\hline
\end{tabular}


Table III. Confusion Matrix

640

\begin{tabular}{|l|l|l|l|}
\cline { 3 - 4 } \multicolumn{2}{c|}{} & \multicolumn{2}{c|}{ Reference } \\
\cline { 3 - 4 } \multicolumn{2}{c|}{} & Target & Non-target \\
\hline \multirow{2}{*}{$\frac{3}{3}$} & Target & True positive & False positive \\
\cline { 2 - 4 } & Non-target & False negative & True negative \\
\hline
\end{tabular}

641

642

Table IV. Performance metrics.

\begin{tabular}{lllll}
\hline $\begin{array}{l}\text { Separation } \\
\text { index }\end{array}$ & $\begin{array}{l}\text { Mean Accuracy } \\
(\boldsymbol{\%})\end{array}$ & $\begin{array}{l}\text { Mean Precision } \\
(\boldsymbol{\%})\end{array}$ & $\begin{array}{l}\text { Mean Recall } \\
(\boldsymbol{\%})\end{array}$ & $\begin{array}{l}\text { Mean F1 } \\
(\boldsymbol{\%})\end{array}$ \\
\hline-0.3 & 43.0 & 49.8 & 45.8 & 47.4 \\
\hline-0.2 & 86.1 & 80.5 & 91.6 & 85.1 \\
\hline-0.1 & 93.9 & 93.5 & 94.4 & 93.9 \\
\hline 0 & 97.0 & 97.0 & 97.2 & 97.0 \\
\hline 0.1 & 98.6 & 98.7 & 98.5 & 98.6 \\
\hline 0.2 & 99.7 & 99.8 & 99.7 & 99.7 \\
\hline 0.3 & 99.98 & 99.97 & 100.00 & 99.98 \\
\hline
\end{tabular}

643

644 


\section{Figure captions}

646 Figure 1. One-dimensional example of the separation index (SI) that measures the magnitude of the 647 gap between two clusters. Vertical lines indicate the lower and upper quantiles of the clusters. The 648 difference between U1 and L2 (numerator) is divided by the difference between L1 and U2

649 (denominator) to calculate the SI value. This method is robust against outliers in between the two

650 clusters that may affect the SI. Figure adapted from (28).

651 Figure 2. Workflow for skew dataset generation. Top panels show scatterplots, bottom panels show 652 density estimates. A) A cluster with a normal distribution is generated, then skew is added through the 653 alpha parameter in the R package $s n$, then the cluster is rescaled. B) Two clusters are combined, then 654 the distances between them can be varied through vector arithmetic operations.

655 Figure 3. Comparison of synthetic and real clusters with representative separation index values 656 ranging from $-0.3,0.0$ to +0.2 , showing overlapping, touching and well-separated clusters,

657 respectively. Top panel shows synthetic data generated from R package clusterGeneration, bottom

658 panel shows real peripheral blood mononuclear cells (PBMCs) data after automated population

659 detection and partition with SPADE3 software followed by separation index calculation.

660 Figure 4. Comparison of synthetic (A) and real (B) flow cytometry data with skewed distributions.

661 Both left-skewed and right-skewed synthetic clusters can be generated that mimic real data.

662 Asymmetry around the mean is clearly shown with contour plots (top) and histograms (bottom).

663 Figure 5. Generation of synthetic two-cluster skewed datasets. Three combinations of cluster pairs are 664 shown here; head-to-head, head-to-tail and tail-to-tail.

665 Figure 6. The synthetic two-cluster separation dataset was run through SPADE3, then the absolute 666 difference between the SPADE3 cell count to the reference value of cluster 1 was calculated. Result 667 demonstrates the accuracy and repeatability of SPADE3 deteriorates as the distance between clusters 668 decreases. Data represents mean \pm 1 SD.

669 Figure 7. The synthetic two-cluster skewed dataset was run through SPADE3, then the gap between 670 the SPADE3 cell population percentages and the reference cell population percentages was calculated. 671 Increasing the cluster skewness in the datasets did not affect SPADE3 performance. However, the 672 accuracy and repeatability of SPADE3 improved as direction of the cluster pairs changed from head673 to-head to head-to-tail, and then to tail-to-tail. All clusters had separation index of -0.2. Data 674 represents mean \pm 1 SD.

675 Figure 8. Performance metrics for SPADE3 analysis of Separation dataset. 


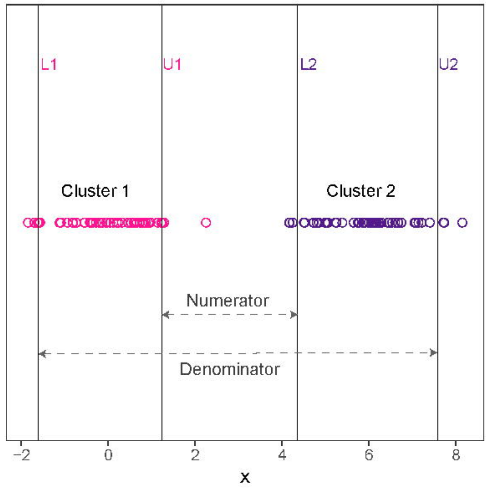


A normal distribution
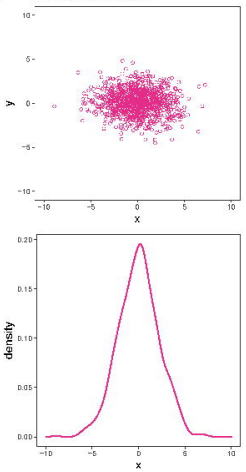

B
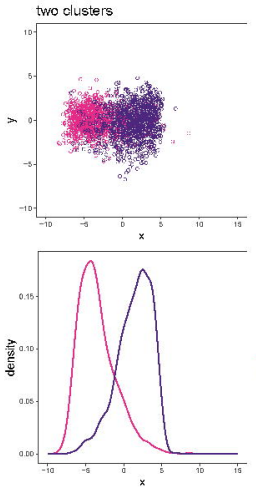

skewed distribution

skewed distribution - rescaled

10.
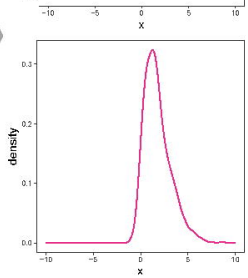

$10-$

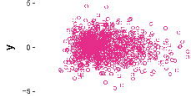

$-10-$
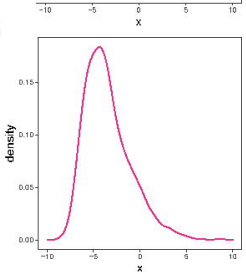

two clusters - shifted

Cluster

- 1

c 2

Cluster

-1
-2

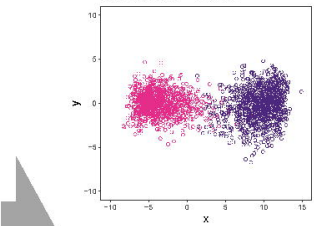

Cluster

101

c. 2

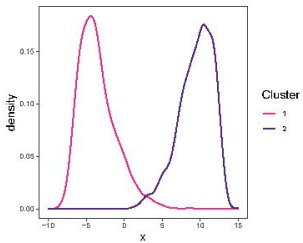




\section{Synthetic data}
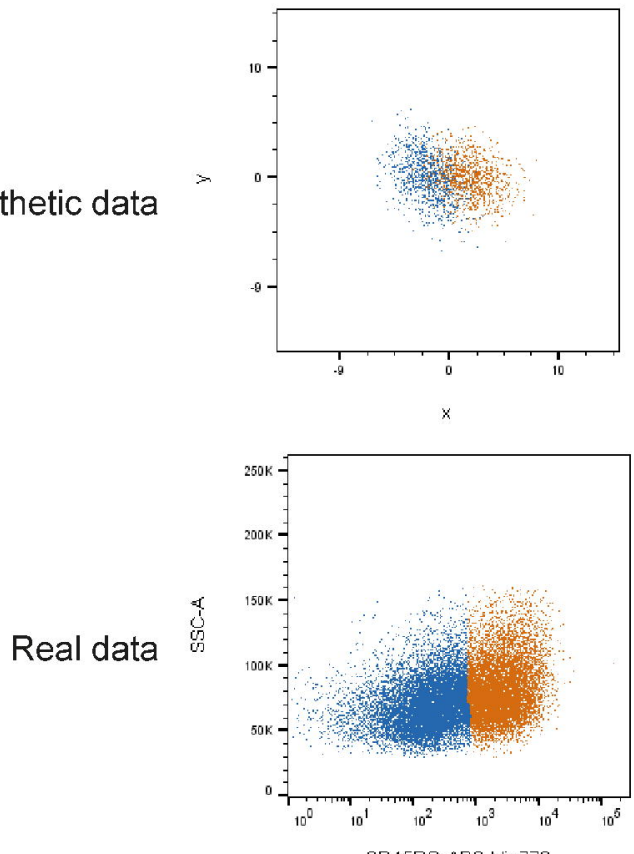
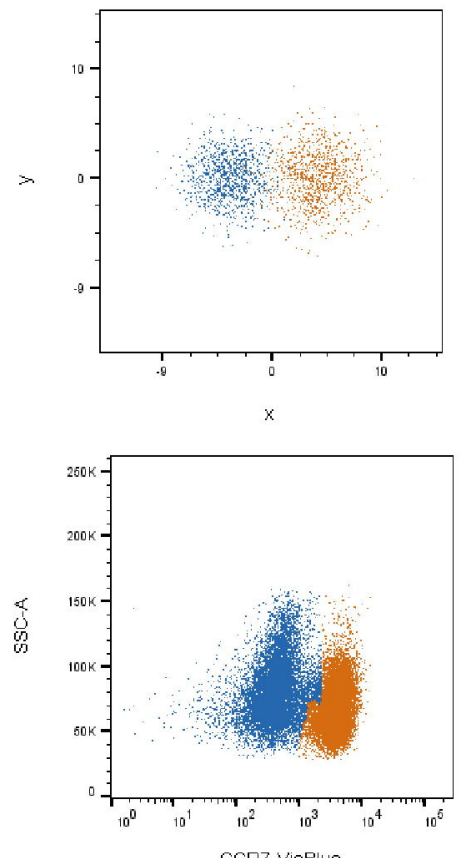
CCR7-VIOB|ШE

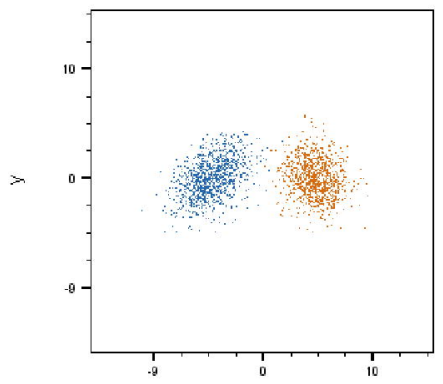

x

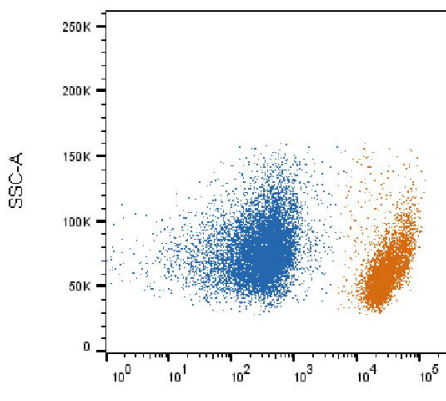

СПА5RП-АРС-VIO770 
(A) Synthetic one-cluster
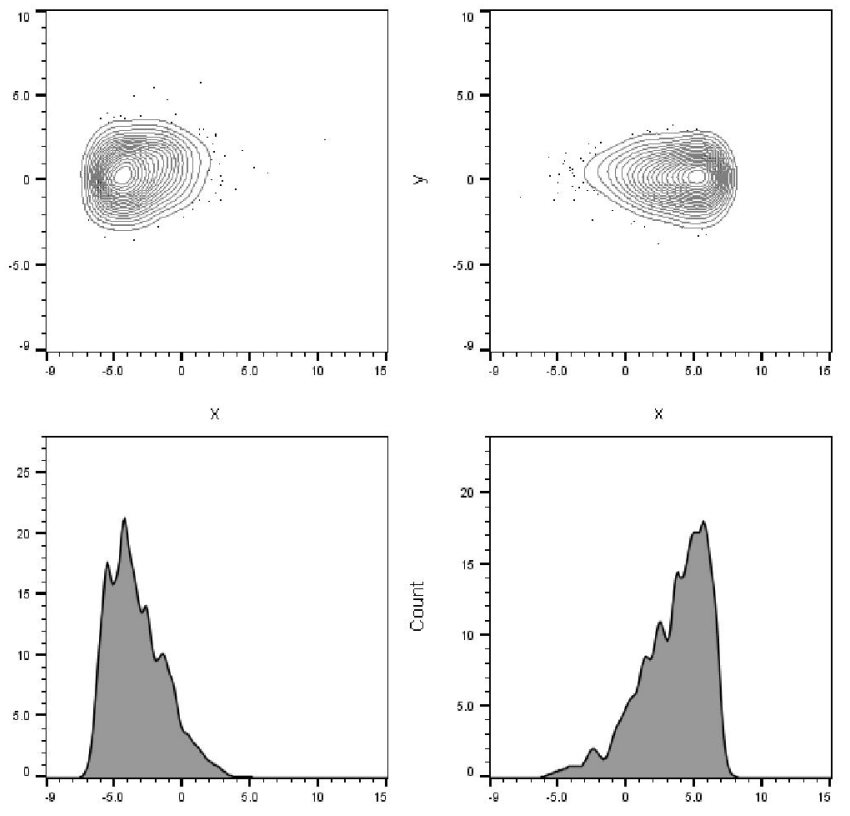

(B) Real one-cluster
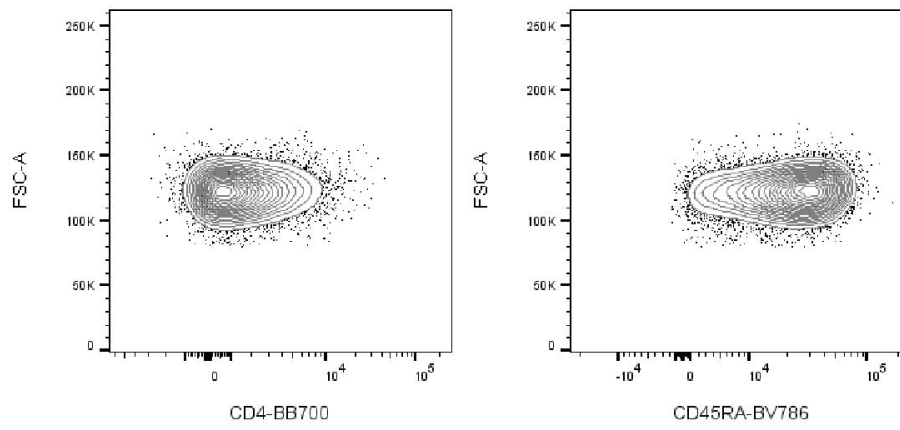

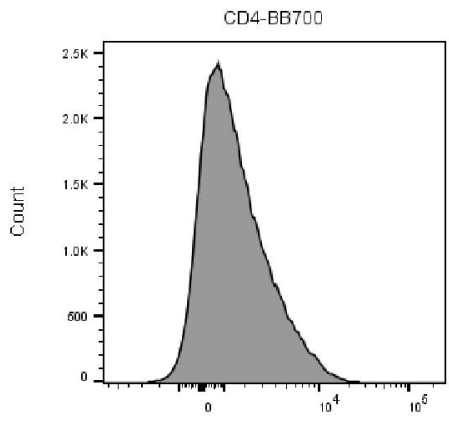

CD4-BR700

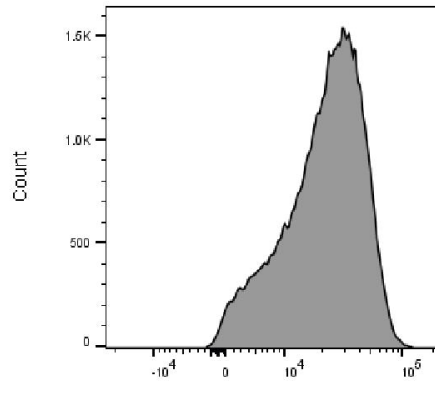



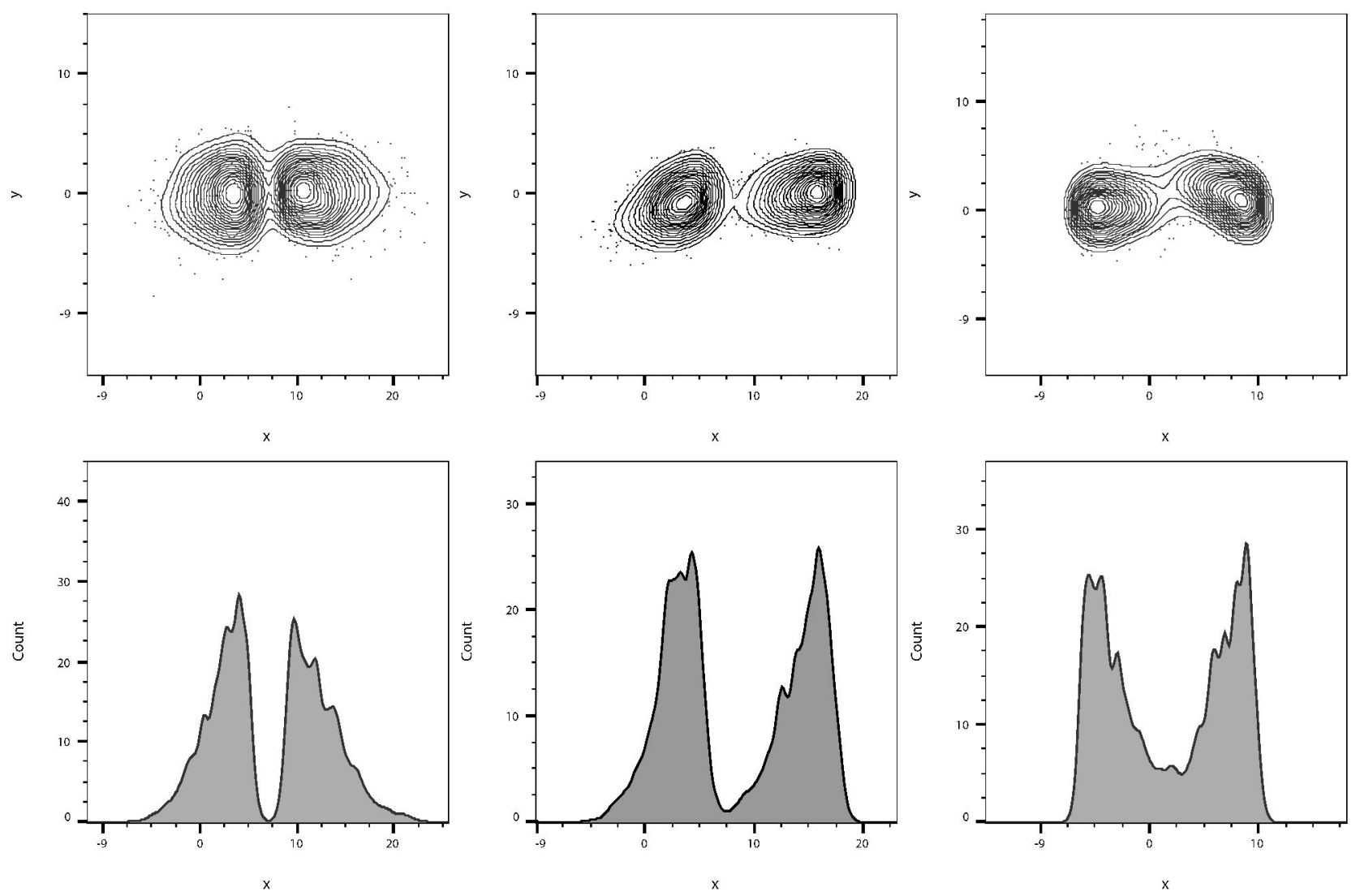


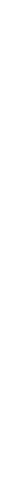




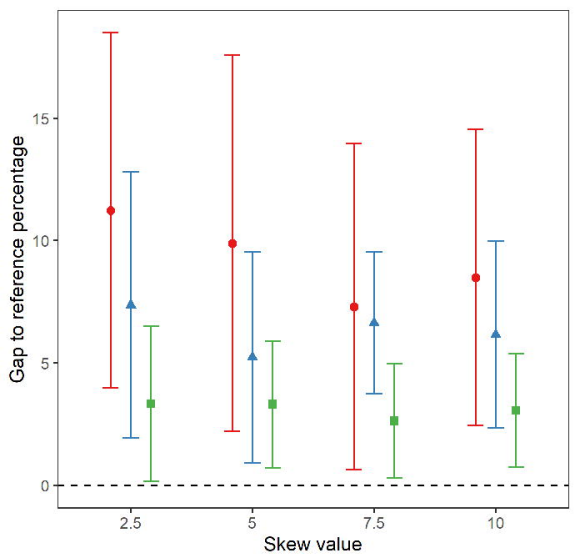

\section{Direction}

- Head-to-head

- Head-to-tail

$\rightarrow$ - Tail-to-tail 


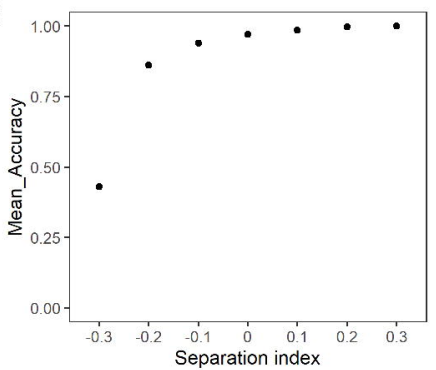

(C)

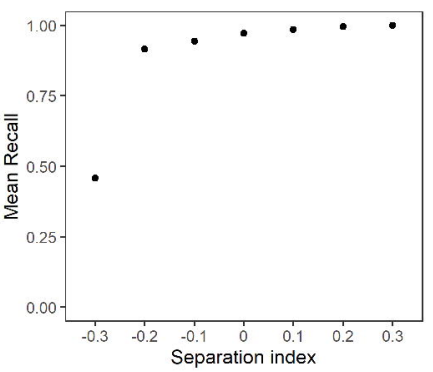

(B)

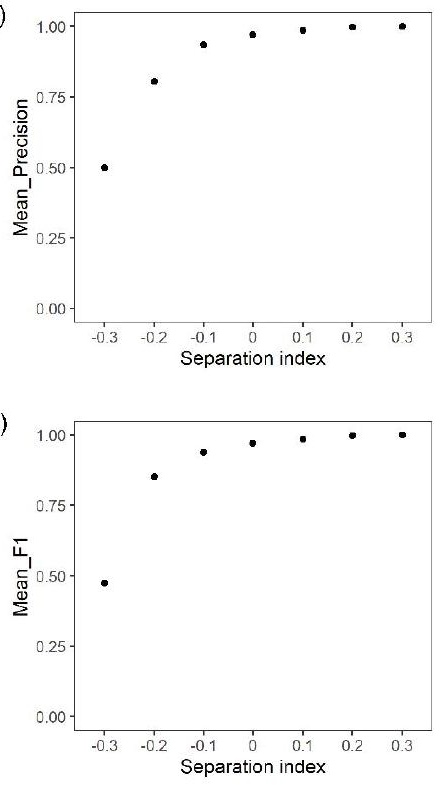

(D)

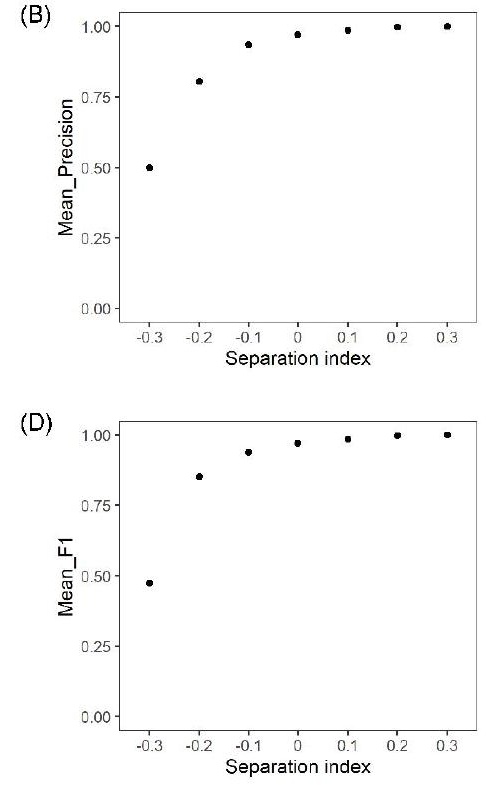

(A)

\section{Separation index}




\section{PDA Journal of Pharmaceutical Science and Technology}

\section{An Authorized User of the electronic PDA Journal of Pharmaceutical Science and Technology (the PDA Journal) is a PDA Member in good standing. Authorized Users are permitted to do the following:}

Search and view the content of the PDA Journal

-Download a single article for the individual use of an Authorized User

-Assemble and distribute links that point to the PDA Journal

-Print individual articles from the PDA Journal for the individual use of an Authorized User

- Make a reasonable number of photocopies of a printed article for the individual use of an

Authorized User or for the use by or distribution to other Authorized Users

\section{Authorized Users are not permitted to do the following:}

Except as mentioned above, allow anyone other than an Authorized User to use or access the PDA Journal

- Display or otherwise make any information from the PDA Journal available to anyone other than an

Authorized User

-Post articles from the PDA Journal on Web sites, either available on the Internet or an Intranet, or in any form of online publications

-Transmit electronically, via e-mail or any other file transfer protocols, any portion of the PDA

Journal

-Create a searchable archive of any portion of the PDA Journal

-Use robots or intelligent agents to access, search and/or systematically download any portion of the PDA Journal

.Sell, re-sell, rent, lease, license, sublicense, assign or otherwise transfer the use of the PDA Journal or its content

-Use or copy the PDA Journal for document delivery, fee-for-service use, or bulk reproduction or distribution of materials in any form, or any substantially similar commercial purpose

-Alter, modify, repackage or adapt any portion of the PDA Journal

- Make any edits or derivative works with respect to any portion of the PDA Journal including any text or graphics

-Delete or remove in any form or format, including on a printed article or photocopy, any copyright information or notice contained in the PDA Journal 